\begin{tabular}{|c|l|}
\hline Title & Periodic Precipitation during Droplet Evaporation on a Substrate \\
\hline Author(s) & Nonomura, Makiko; Kobay ashi, Ryo; Nishiura, Y asumasa; Shimomura, Masatsugu \\
\hline Citation & Journal of the Physics Society Japan, 72(10), 2468-2471 \\
\hline https://doi.org/10.1143/PSJ.72.2468 \\
\hline Issue Date & 2003-10 \\
\hline Doc URL & http://hdl.handle.net/2115/40064 \\
\hline Rights & ○ 2003 The Physical Society of Japan \\
\hline Type & article (author version) \\
\hline File Information & nishiura 40.pdf \\
\hline
\end{tabular}

Instructions for use 


\title{
Periodic Precipitation during Droplet Evaporation on a Substrate
}

\author{
Makiko Nonomura $^{1}{ }^{*}$, Ryo Kobayashi $^{2}$, Yasumasa Nishiura ${ }^{2}$ and Masatsugu Shimomura ${ }^{2,3}$ \\ ${ }^{1}$ Department of Mathematical and Life Sciences, Graduate School of Science, Hiroshima University, \\ Higashi-Hiroshima 739-8526, Japan \\ ${ }^{2}$ Research Institute for Electronic Science, Hokkaido University, Sapporo 060-0812, Japan \\ ${ }^{3}$ Dissipative-Hierarchy Structures Laboratory, Spatio-Temporal Function Materials Research Group, \\ Frontier Research System, RIKEN, Wako 351-0198, Japan
}

(Received December 7, 2009)

\begin{abstract}
We present a model of motion and evaporation of a droplet of a solute along with precipitation. It is demonstrated that the model can simulate the formation of stripe patterns parallel to the droplet edge resulting from periodic precipitation.
\end{abstract}

KEYWORDS: periodic precipitation, evaporation, patterning, supersaturation

\section{Introduction}

Recently, mesoscopic pattern formations in dissipative systems have engaged the interest of the engineering community. Among them, evaporation of a droplet on a solid substrate is a typical phenomenon. There has been some experimental research on this type of pattern formation. ${ }^{1,2}$ It has been reported that the stripes parallel to the contact line are observed under some conditions, while stripes perpendicular to the contact line are obtained under other conditions. Also, patterns of small drops located in a regular lattice, or more complicated patterns have been found. Although this phenomenon seems to be simple, it includes several complex processes, for example, evaporation, convection and precipitation.

There are some related theories of interest. The effects of flow of fluids inside the droplet has been studied by Fischer. ${ }^{3}$ In ref. 4, Adachi et al. explained the oscillating motion of the contact line as competition between the interfacial tension and the friction which the contact line is subjected to when particles flow into the droplet boundary. The dynamics of an evaporating wetting liquid film in the presence of dissolved surfactant has been investigated by Danov et al..$^{5}$

However, to the authors' knowledge, there are no models which describe pattern formation during evaporation of a droplet. The purpose of the present study is to derive a set of equations for such a system using the local volumes of the solution and the solutes from the Navier-Stokes equation in the lubrication approximation, ${ }^{6,7}$ and to carry out numerical simulations.

In this article, a model of the motion of an evaporating liquid droplet on a substrate is

\footnotetext{
${ }^{*}$ E-mail address: nonomura@math.sci.hiroshima-u.ac.jp
} 
presented first, then it is extended to a model which includes a precipitation process. We concentrate on the formation of the stripe pattern parallel to the contact line, which is a product of the periodic precipitation; thus our model is one-dimensional.

\section{Modeling of the Motion of a Droplet}

We assume that the liquid is a Newtonian viscous fluid of viscosity $\eta$. The thickness of the liquid film is denoted by $h(\boldsymbol{r}, t)$ where $\boldsymbol{r}=(x, y)$, as shown in Fig. 1 . Thus $h$ is a non-negative variable and $h>0$ implies that the surface of the substrate is wet, while $h=0$ means it is dry.

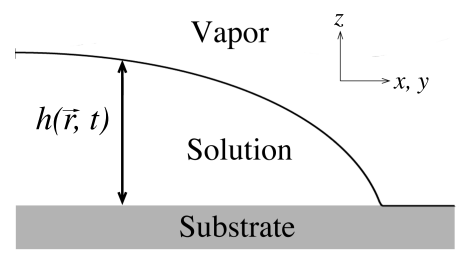

Fig. 1. The physical configuration showing the Cartesian coordinate system and describing an evaporating thin liquid film on a solid substrate.

The transport equation of an incompressible solution is

$$
\frac{\partial h}{\partial t}=-\nabla \cdot(h \boldsymbol{v})
$$

where $\boldsymbol{\nabla}$ is a nabla operator with respect to $\boldsymbol{r}=(x, y)$, and $\boldsymbol{v}(\boldsymbol{r}, t)$ is the vertically averaged liquid velocity. Applying the lubrication approximation to a viscous-liquid flow, ${ }^{6,7}$ the averaged velocity $\boldsymbol{v}$ is given by

$$
\boldsymbol{v}=-\frac{1}{3 \eta} h^{2} \nabla \frac{\delta F}{\delta h}
$$

where $F[h]$ is a free energy functional. By taking the surface energy and the gravity into account, we set the form of $F[h]$ to be,

$$
F[h]=\int d \boldsymbol{r}\left[\gamma_{l v} \sqrt{1+(\boldsymbol{\nabla} h)^{2}} \chi(h)+\gamma_{s l} \chi(h)+\gamma_{v s}(1-\chi(h))+\frac{\rho g}{2} h^{2}\right],
$$

where $\rho$ is the density of the solution and $g$ is the gravitational acceleration. The function $\chi(h)$ is given by

$$
\chi(h)= \begin{cases}1 & h>0 \\ 0 & h \leq 0,\end{cases}
$$

thus $\chi(h)$ is an indicator of the wet region. For simplicity, we assume that $\rho$ is independent of the concentration of solutes. The coefficients $\gamma_{l v}, \gamma_{v s}$ and $\gamma_{s l}$ denote surface energy densities of the liquid/vapor interface, vapor/solid interface and solid/liquid interface, respectively, and 
they are also assumed to be constant. The functional derivative of $F[h]$ is calculated as follows:

$$
\frac{\delta F}{\delta h}=-\gamma_{l v} \chi(h) \nabla \cdot\left(\frac{\nabla h}{\sqrt{1+(\boldsymbol{\nabla} h)^{2}}}\right)+\chi^{\prime}(h)\left(\gamma_{l v} \frac{1}{\sqrt{1+(\boldsymbol{\nabla} h)^{2}}}+\gamma_{s l}-\gamma_{v s}\right)+\rho g h .
$$

Note that the second term of the right-hand side of eq. (5) includes a singularity since $\chi^{\prime}(h)$ is Dirac's delta function. The equilibrium condition under the constant-volume constraint is $\frac{\delta F}{\delta h}=C$ with some constant $C$, and it yields the following two equations:

$$
-\gamma_{l v} \nabla \cdot\left(\frac{\nabla h}{\sqrt{1+(\nabla h)^{2}}}\right)+\rho g h=C
$$

in the wet region where $h>0$, and

$$
\gamma_{l v} \frac{1}{\sqrt{1+(\nabla h)^{2}}}+\gamma_{s l}-\gamma_{v s}=0
$$

at the contact line (the boundary of the wet region). Equation (6) implies that the profile of the droplet on the substrate is determined by the balance between surface tension and gravity, since the expression $\boldsymbol{\nabla} \cdot\left(\nabla h / \sqrt{1+(\nabla h)^{2}}\right)$ gives the mean curvature of the surface $z=h$. In particular, the surface of the droplet has a constant mean curvature in the absence of gravity, which is consistent with the minimal surface theory. Equation (7) is nothing but the Young $\AA]$ Dupré equation $\gamma_{l v} \cos \theta+\gamma_{s l}-\gamma_{v s}=0$ for the contact angle $\theta$, since $\tan \theta=|\nabla h|$ holds at the contact line.

Using eqs. (1), (2) and (5) and taking the evaporation process into account, we derive

$$
\begin{aligned}
\frac{\partial h}{\partial t}=\boldsymbol{\nabla} \cdot\left\{\frac { 1 } { 3 \eta } h ^ { 3 } \boldsymbol { \nabla } \left[-\gamma_{l v} \chi(h) \boldsymbol{\nabla}\right.\right. & \cdot\left(\frac{\boldsymbol{\nabla} h}{\sqrt{1+(\boldsymbol{\nabla} h)^{2}}}\right) \\
& \left.\left.+\chi^{\prime}(h)\left(\gamma_{l v} \frac{1}{\sqrt{1+(\boldsymbol{\nabla} h)^{2}}}+\gamma_{s l}-\gamma_{v s}\right)+\rho g h\right]\right\}-f_{e}
\end{aligned}
$$

where $f_{e}$ denotes the evaporation rate. The dimensionless version of eq. (8) is obtained by nondimensionalizing the thickness and the space variables by the characteristic thickness $h_{0}$ and the time variable by $t_{0}=\frac{\eta}{h_{0} \rho g}$ as follows:

$$
\begin{array}{r}
\frac{\partial h}{\partial t}=\nabla \cdot\left\{\frac { 1 } { 3 } h ^ { 3 } \boldsymbol { \nabla } \left[-\gamma \chi(h) \nabla \cdot\left(\frac{\nabla h}{\sqrt{1+(\boldsymbol{\nabla} h)^{2}}}\right)\right.\right. \\
\left.\left.+\chi^{\prime}(h)\left(\gamma \frac{1}{\sqrt{1+(\boldsymbol{\nabla} h)^{2}}}-\Gamma\right)+h\right]\right\}-f_{e}
\end{array}
$$

where $\gamma=\frac{\gamma_{l v}}{\rho g h_{0}^{2}}$ and $\Gamma=\frac{\gamma_{v s}-\gamma_{s l}}{\rho g h_{0}^{2}}$. Note that the dimensionless variables $h, \boldsymbol{r}$ and $t$ as well as the evaporation rate $f_{e}$ are written in the same form as the original expressions with dimensions in eq. (8). Evaporation is caused by a vapor pressure deviation from equilibrium. We give the evaporation rate in the form $f_{e}=e\left[p_{0} \exp (A \Phi)-p_{v}\right]$ where $e$ is a dimensionless con- 
stant and $\Phi=-\gamma \chi(h) \nabla \cdot\left(\boldsymbol{\nabla} h / \sqrt{1+(\boldsymbol{\nabla} h)^{2}}\right)+\chi^{\prime}(h)\left(\gamma / \sqrt{1+(\boldsymbol{\nabla} h)^{2}}-\Gamma\right)$. The parameter $p_{0}$ indicates the dimensionless equlibrium vapor pressure for a flat surface of bulk liquid and $p_{v}$ is the dimensionless pressure in the vapor phase. The dimensionless constant $A$ is inversely proportional to the absolute temperature. Similar forms for evaporation were obtained by Padmakar et al. ${ }^{8}$ and Wayner. ${ }^{9}$ For simplicity, we set $p_{0}=1.0$ and $p_{v}=0.0$.

In the following simulations, the indicator function $\chi(h)$ is approximated by the function

$$
\chi_{\delta}(h)= \begin{cases}\tanh (h / \delta) & h>0 \\ 0 & h \leq 0,\end{cases}
$$

where $\delta$ is a small positive number. If the relation $\gamma>\Gamma$ holds and the slope of $h$ is small enough, i.e., $|\nabla h| \ll 1$, this approximation leads to the kinetic equation which is almost equivalent to the model equation presented in refs. 10-12.

One-dimensional simulations of the evolution equation (9) are carried out, and the results are presented in Fig. 2 and Fig. 3. We imposed a no flux condition and the Neumann boundary condition on each end, and the initial data were given as $h(x)=\max \left(h_{l}\left(1-(x / 2.5)^{2}\right), 0\right)$, where the system size is 4 . The coefficients $\delta$ and $\gamma$ were fixed to the values $\delta=0.010$ and $\gamma=2.0$, respectively.

Curves drawn in Fig. 2 indicate the equilibrium profiles of droplets obtained after a sufficiently long evolution without evaporation. In the simulations shown in Fig. 2(a), we adopted the same initial data and controlled the value of the parameter $\Gamma$. On the other hand, in Fig. $2(\mathrm{~b})$, the parameter $\Gamma$ was fixed and the initial data (or the total volume) were controlled in the simulations.

The evaporation processes are presented in Fig. 3, where initial data are taken to be a stationary solution for $e=0.0$ indicated for the largest droplet in Fig. 2 (b). The parameter $e$ is set to be $2.5 \times 10^{-3}$, and the computation is performed until the whole droplet completely evaporates. When the parameter $A$ becomes large, evaporation near the edge becomes marked.

\section{Modeling for a Solution}

In this section, we extend the droplet model (9) to a set of evolution equations for a solution which includes the precipitation process. Let us take the variables $s(\boldsymbol{r}, t)$ and $q(\boldsymbol{r}, t)$ to be the local amount of solutes and precipitates, respectively. We assume the local concentration of solutes does not depend on the $z$-direction. Expressing the generation rate of precipitate by an adequate function $G(h, s, q)$, we have

$$
\begin{aligned}
& \frac{\partial s}{\partial t}=-\boldsymbol{\nabla} \cdot(s \boldsymbol{v})+D_{s} \boldsymbol{\nabla} \cdot\left[h \boldsymbol{\nabla}\left(\frac{s}{h}\right)\right]-G(h, s, q) \\
& \frac{\partial q}{\partial t}=\boldsymbol{\nabla} \cdot\left[\hat{D}_{q} \boldsymbol{\nabla} q\right]+G(h, s, q),
\end{aligned}
$$



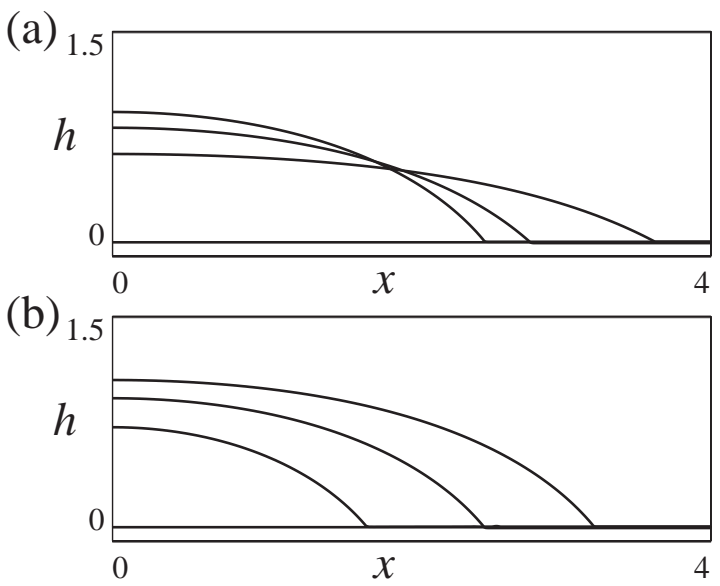

Fig. 2. The profiles of droplets $z=h$ in the stationary states. (a) $\Gamma=0.20,0.90,1.6$ and $h_{l}=1.0$. (b) $\Gamma=0.20$ and $h_{l}=0.50,1.0,1.5$. In the simulations, the spatial mesh size and the temporal mesh size were taken to be 0.010 and $5.0 \times 10^{-4}$, respectively.
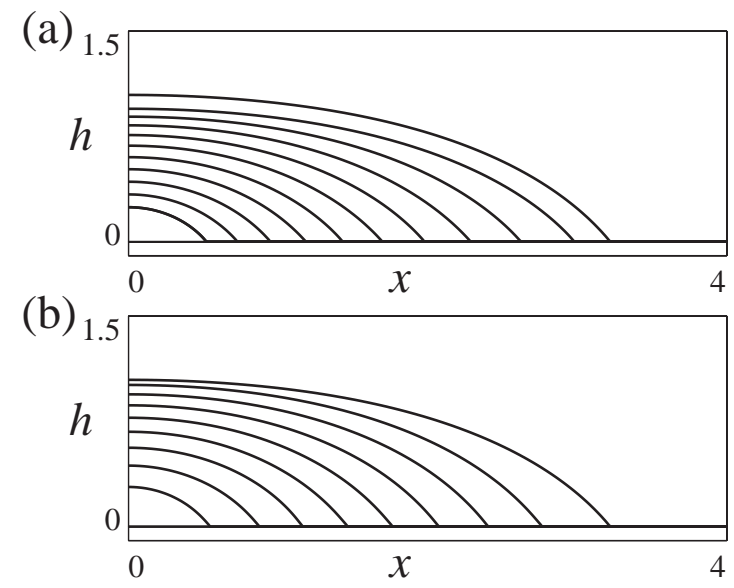

Fig. 3. Evaporation process with $e=2.5 \times 10^{-3}$. In this simulation, the spatial mesh size and the temporal mesh size were taken to be 0.010 and $5.0 \times 10^{-4}$, respectively. The curves in (a) and (b) represent the profile of $h$ every 50 unit times for (a) $A=0.0$ and (b) $A=0.060$.

where $\boldsymbol{v}$ is given by eq. (2) and $D_{s}$ is a diffusion coefficient for $s$ in the solution. We assume that the diffusion term for $q$ is written in the form $\hat{D}_{q}(h, s)=D_{q} \chi(h)\left[1+\tanh \left(\left(s-h c_{1}\right) / \delta_{q}\right)\right] / 2$, where $D_{q}$ is a positive constant and $\delta_{q}$ is a small number. Note that the total amount of $s+q$ is conserved.

We take the form of the function $G(h, s, q)$ to be

$$
G(h, s, q)= \begin{cases}\omega_{1} q\left(s-h c_{1}\right) & s<h c_{1} \\ \omega_{2} s(q-k(h, s)) & s \geq h c_{1}, q \geq k(h, s) \\ \omega_{3} q(q-k(h, s)) & s \geq h c_{1}, q<k(h, s),\end{cases}
$$


where $\omega_{i}$ 's are positive constants and the function $k(h, s)$ is as shown in Fig. 4 , where $c_{1}$ is a solubility concentration and $c_{2}$ a critical concentration above which no supersaturated solution can exist.

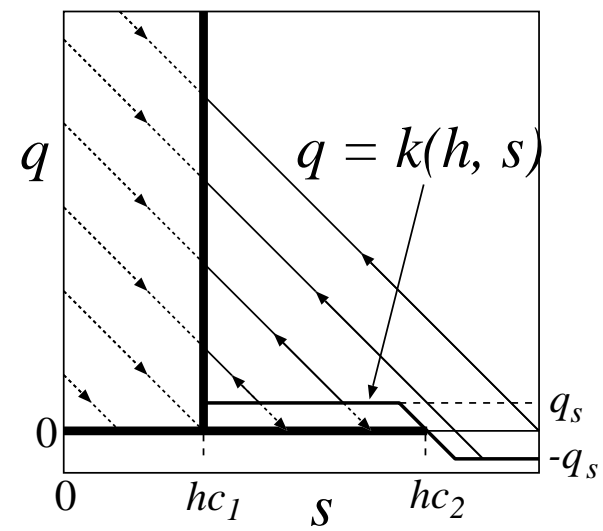

Fig. 4. Local dynamics in the $s-q$ plane for the fixed value of $h$ is displayed. The solid arrows indicates precipitation from supersaturated solution and the dotted one implies dissolution. The bold lines correspond to the stable $\left(s \leq h c_{1}\right)$ or metastable $\left(s>h c_{1}\right)$ states. The graph of $q=k(h, s)$ is designed for a separatrix of the flow in the supersaturated region, where $q_{s}$ is taken to be small.

An amount $s$ larger than $h c_{1}$ implies the state of supersaturation. When $s$ surpasses $h c_{2}$, precipitation occurs. Evaporation of solvent increases the solute concentration, particularly near the front.

In addition, in order to describe the self-pinning observed in the experiments, ${ }^{2}$ we consider the modification of the substrate by the precipitates, by taking $\Gamma$ as a function of $q, \hat{\Gamma}(q)$. We assume $\hat{\Gamma}(q)=\Gamma+\Gamma_{q} \tanh \left(q / \delta_{g}\right)$ where $\Gamma$ and $\Gamma_{q}$ are constants and $\delta_{g}$ is a small number. If the substrate covered by precipitates has a higher affinity to the solution than a noncovered substrate, a positive constant must be chosen to be $\Gamma_{q}$.

We carry out one-dimensional simulations for the set of eqs. (9), (11) and (12). The parameters are chosen to be $A=0.060, \Gamma=0.20, D_{s}=0.025, c_{1}=0.30, c_{2}=0.60, \omega_{1}=$ $\omega_{2}=\omega_{3}=20, \delta_{g}=0.10$ and $D_{q}=1.0 \times 10^{-5}$. We use the data indicated for the largest droplet in Fig. 2 (b) as the initial data $h(x, 0)$. The initial condition for $s$ is provided by $s(x, 0)=0.20 \cdot h(x, 0)$.

Figure 5 shows the deposit for different values of $\Gamma_{q}$. Figures $5(\mathrm{a})-5(\mathrm{c})$ show the profiles of $q$. The droplet radius versus time is shown in Fig. 6. The dotted and solid curves correspond to Figs. 5(a) and 5(b), respectively. As shown in Fig. 6, the radius with $\Gamma_{q}=0.0$ monotonically decreases, whereas in case of $\Gamma_{q} \neq 0.0$, the oscillating recession of the droplet edge takes place in cooperation with precipitation near the edge. Figure 7 summarizes the dependence of precipitation on the evaporation rate. 

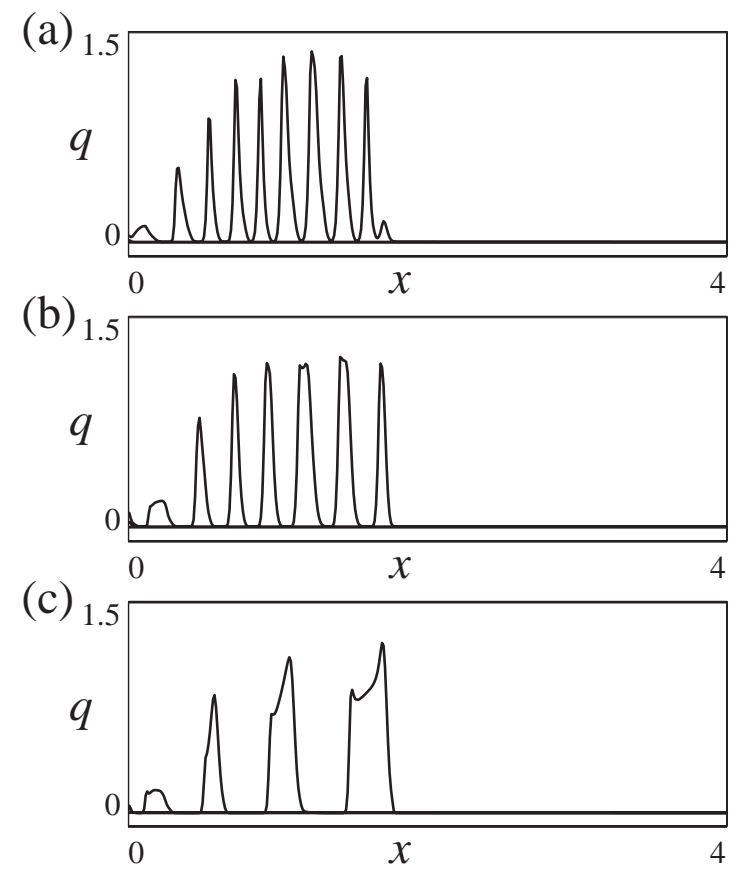

Fig. 5. The profiles of precipitation $q$ for $\Gamma_{q}$ of (a) 0.0, (b) 0.25 and (c) 1.0. In the simulations, the spatial mesh size and the temporal mesh size were taken to be 0.010 and $5.0 \times 10^{-4}$, respectively. Evaporation rates are set as $e=2.5 \times 10^{-3}$ and $A=0.060$.

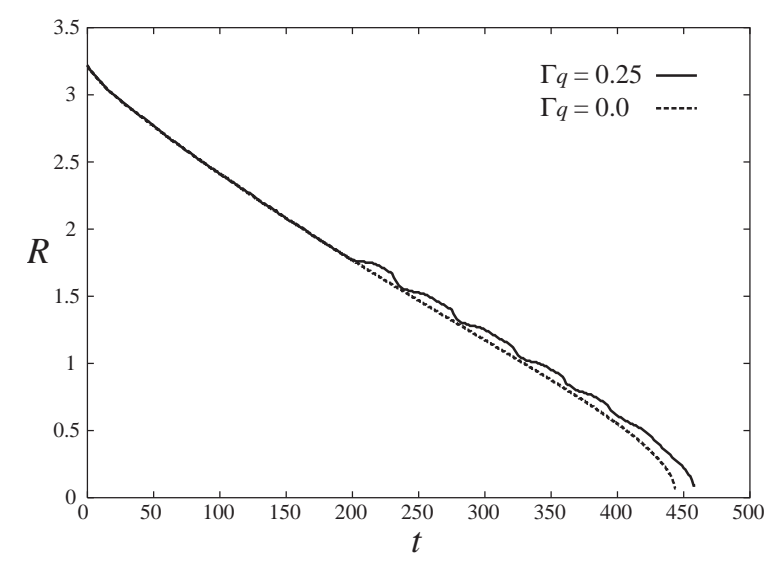

Fig. 6. Radius of droplet vs time. The dotted and solid curves correspond to Figs. 5(a) and 5(b), respectively.

\section{Conclusion}

We proposed a model of periodic precipitation from a droplet of solution caused by evaporation. In our simulations, precipitation takes place only in the vicinity of the contact line except in the very final stage of evaporation, as was observed in experiments. Our model could reproduce striped patterns because supersaturation was taken into account $\left(c_{1}<c_{2}\right)$, which is 

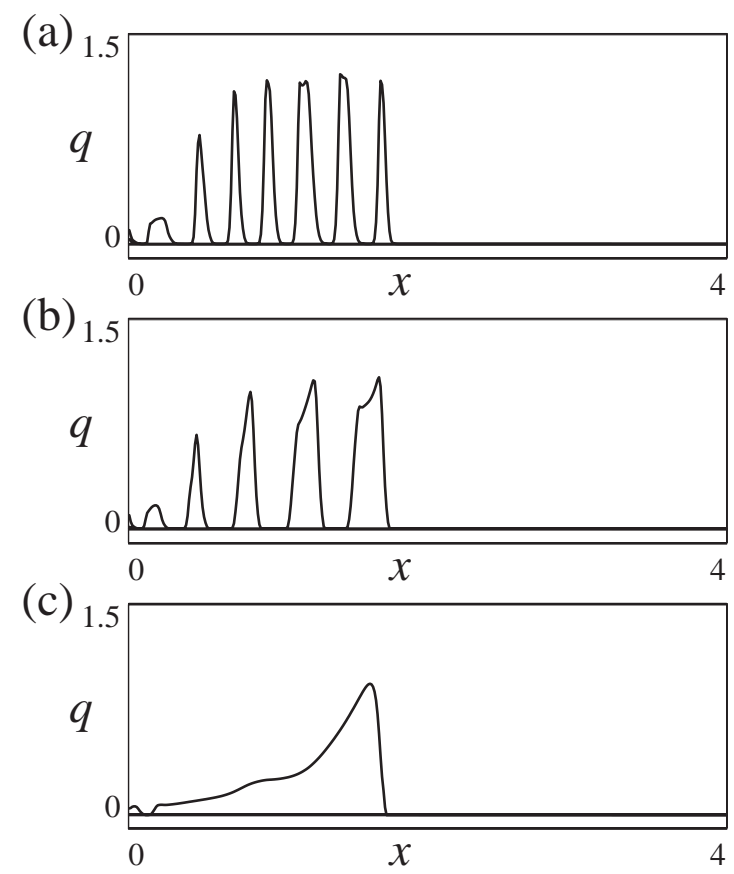

Fig. 7. The profiles of precipitation $q$ with (a) $e=2.50 \times 10^{-3}$, (b) $e=1.25 \times 10^{-3}$ and (c) $e=$ $2.50 \times 10^{-4}$. In the simulations, the spatial mesh size and the temporal mesh size were taken to be 0.010 and $5.0 \times 10^{-4}$, respectively. Other parameters were set to be $\Gamma_{q}=0.25$ and $A=0.060$.

essential for this kind of periodic precipitation. Actually, the pattern becomes irregular in the absence of supersaturation $\left(c_{1}=c_{2}\right)$, since the precipitation occurs not only near the contact line but also inside the droplet.

The oscillating recession of the droplet edge also could be reproduced by introducing the dependence of the affinity of solution $\Gamma_{q}$ on the density of the precipitate $q$, which changed the period of precipitation, as shown in Fig. 5.

In this study, we concentrated on the periodic precipitation, which is essentially a onedimensional phenomenon, and did not consider the formation of two-dimensional patterns, such as dotted arrays and stripes parallel to the receding direction. We are trying to extend our model to a two-dimensional one, and it will be given elsewhere together with further simulations. 


\section{References}

1) O. Karthaus, L. Grasjo, N. Maruyama and M. Shimomura: Chaos 9 (1999) 308.

2) R. D. Deegan: Phys. Rev. E 61 (2000) 475.

3) B. J. Fischer: Langmuir 18 (2002) 60.

4) E. Adachi, A. S. Dimitrov and K. Nagayama: Langmuir 11 (1995) 1057.

5) K. D. Danov, N. Alleborn, H. Raszillier and F. Durst: Phys. Fluids 10 (1998) 131.

6) A. Oron, S. H. Davis and S. G. Bankoff: Rev. Mod. Phys. 69 (1997) 931.

7) M. B. Williams and S. H. Davis: J. Colloid Interface Sci. 90 (1982) 220.

8) A. S. Padmakar, K. Kargupta and A. Sharma: J. Chem. Phys. 110 (1999) 1735.

9) P. C. Wayner: Langmuir 9 (1993) 294.

10) L. M. Pismen and Y. Pomeau: Phys. Rev. E 62 (2000) 2480.

11) L. Brusch, H. Kühne, U. Thiele and M. Bär: Phys. Rev. E 66 (2002) 011602.

12) U. Thiele, M. G. Velarde, K. Neuffer and Y. Pomeau: Phys. Rev. E 64 (2001) 031602. 